Fecha de recepción: abril 2020 Fecha de aceptación: mayo 2020 Versión final: junio 2020

\section{Los coros infantiles y juveniles en el GBA. Reflexiones en torno a los cambios de las políticas culturales en la gestión pública ${ }^{1}$}

Verónica Talellis ${ }^{(1)}$ y Eugenia Amantía ${ }^{(2)}$

\begin{abstract}
Resumen: Desde fines del siglo XX en Argentina comienzan a multiplicarse propuestas que instalan al arte como herramienta para la inclusión social. En consecuencia diversos autores han orientado sus investigaciones reflexionando acerca del lugar del "arte" y su dimensión social en un grupo o comunidad. Siguiendo esta línea, nuestras indagaciones hacen foco en los coros infantiles y juveniles del Gran Buenos Aires, gestionados por el Estado. A partir del relevamiento realizado en el período 2016-2018 nos proponemos reflexionar, a partir de una perspectiva descriptiva-comparativa de los datos relevados, sobre algunos aspectos que se vislumbran claves en la disputa por los sentidos del hacer, implicados en los diseños de políticas culturales de cada gestión.
\end{abstract}

Palabras clave: coros infantiles y juveniles - métodos de enseñanzas - inclusión social gestiòn pública - diseño cultural

[Resúmenes en inglés y portugués en las páginas 54 - 55]

(1) Profesora y Licenciada en Ciencias Antropológica (UBA). Es investigadora de temáticas relacionadas con la música como expresión artística para la transformación social y forma parte del proyecto de reconocimiento institucional avalado por la FFyL-UBA “Políticas Arte transformadoras. Usos del arte para la transformación social en la ciudad de Buenos Aires", dirigido por la Dra. Julieta Infantino. Integra el grupo Música e Inclusión con el cual desarrolla el Proyecto de investigación, Agregando Valor 2019.

(2) Técnica en Dirección de Coros y Orquestas Infantiles y Juveniles (UNDAV). Es docente de música e investigadora en temáticas relacionadas a la actividad coral infantojuvenil y al cancionero escolar. Integra el grupo Música e Inclusión con el cual desarrolla el Proyecto de investigación sobre las orquestas y coros infantiles y juveniles del Gran Buenos Aires radicado en la Universidad Nacional de Avellaneda (PROAPI 2014, UNDAVCYT 20172019. Agregando Valor 2019). 


\section{Introducción}

A finales de la década de 1990 y con mayor intensidad desde inicios del 2000 en Argentina comienzan a visibilizarse propuestas que instalan al arte como herramienta para la inclusión social. Diversos autores han orientado sus investigaciones reflexionando sobre las actividades artísticas-educativas y su dimensión social en un determinado grupo o comunidad. En consecuencia, distintos estudios han indagado sobre el lugar de las "artes" como herramienta de intervención para la inclusión, integración y/o transformación social (Avenburg, Cibea y Talellis, 2017, 2019; Infantino, 2008 y 2011; Mercado, 2019; Moyano, 2019, entre otros). A partir del análisis de proyectos que proponen distintos lenguajes artísticos, estas investigaciones coinciden en señalar diversas maneras en que estas propuestas apuntan a la enseñanza de una disciplina artística como herramienta para potenciar la transformación de la vida cotidiana de quienes la practican.

Siguiendo esta línea, iniciamos nuestras primeras indagaciones sobre los proyectos de coros infantiles y juveniles, los cuales se desarrollan en la mayoría de los casos como actividades paralelas a proyectos de orquestas, siendo en algunas ocasiones soportes de la actividad orquestal y en otras coros "per se". Al igual que las iniciativas de orquestas a las que hacemos referencia en otros trabajos (Avenburg, Cibea y Talellis, 2017; Talellis, 2019), estos proyectos se caracterizan por dirigir sus acciones al trabajo con niños, niñas y jóvenes que viven en contextos mayoritariamente precarizados. Al tiempo que coinciden en poner el acento en la enseñanza musical a través de la práctica coral, presentan algunas discrepancias en sus modalidades de implementación.

Si bien este tipo de propuestas se han multiplicado a nivel nacional en este último tiempo, el número total de coros con estas características se encuentra poco visibilizado.

Tomando como eje bisagra el cambio de Gobierno ocurrido en el 2015 tanto a nivel Nacional, Provincial como Municipal2 ${ }^{2}$, en el 2016 comenzamos un relevamiento de estos proyectos que se continuó hasta el 2019. Cabe aclarar que para el presente trabajo nos basamos en los datos relevados durante el período 2016-2018. Partiendo de una perspectiva que combina un abordaje descriptivo con el enfoque etnográfico, nos proponemos abordar comparativamente el análisis de los datos relevados sobre algunos aspectos claves en la disputa por los sentidos del hacer e implicados en los diseños de políticas culturales implementadas por las distintas gestiones pública.

Algunos interrogantes que motivaron la presente investigación fueron:

¿Cómo se dan los cambios entre las administraciones públicas que se suceden? ¿Qué variables se deben tener en cuenta para analizar los procesos transicionales de un cambio de administración a otra en el ámbito estatal, respondan estas al mismo partido del Gobierno electo o no? En el caso concreto de los proyectos que nos ocupan aquí nos preguntamos: ¿Se continuaron, se desmantelaron o se modificaron? En este sentido, entendemos que tanto la planificación como la puesta en práctica de estas iniciativas en cada gestión se configuraran en función de las expectativas y las experiencias de los destinatarios. Asimismo, cabe señalar que esta dinámica articulada con los lineamientos de las políticas públicas coyunturales generará variaciones, tanto entre el diseño y la acción en un mismo proyecto, como entre diferentes proyectos. 
Si bien son escasos los estudios que se aboquen específicamente a los coros y la inclusión social, cabe señalar, no obstante, que existen -con mayor crecimiento en estos últimos años- unas pocas investigaciones sobre temáticas afines (ver por ejemplo Lenger, 2013), junto con actividades que proponen espacios para reflexionar y repensar la práctica coral y su conexión con los desarrollos socioculturales. Ejemplo de ello son algunas de las mesas presentadas en el $1^{\circ}$ Congreso Coral Argentino de OFADAC en 2013, las asociaciones de directores corales como ADICORA y las redes corales como la Red Coral Argentina.

Respecto a la administración pública, hemos identificado -hasta el momento- tres propuestas gubernamentales que se involucran con esta temática. Una a nivel Nacional, enmarcada dentro del Programa Nacional de Orquestas y Coros del Bicentenario del Ministerio de Educación de la Nación; otra denominada "Orquestas y Coros para la Equidad", que se desarrolla a nivel local, dependiente de la gerencia operativa "Música para la equidad" (Ministerio de Educación de la Ciudad de Buenos Aires) y la tercera denominada "Programa Provincial de Orquesta Escuela", dependiente de la provincia de Buenos Aires. El objetivo del presente trabajo es compartir una serie de reflexiones surgidas a partir del relevamiento realizado durante el periodo ya mencionado en el marco de la investigación que venimos desarrollando sobre los proyectos de orquestas y coros del GBA con el Grupo de Investigación sobre Música e inclusión (GIMI) ${ }^{3}$.

Dada la falta de una sistematización de los datos existentes, buscamos en primer lugar mapear el complejo escenario de estas propuestas en el GBA al momento de transición de una gestión a otra (a fines de 2015 cambia el Gobierno en sus tres niveles: Nacional, Provincial y Municipal); en segundo lugar nos proponemos reflexionar sobre algunos aspectos que se vislumbran claves en la disputa por los sentidos del hacer, implicados en las direcciones que toman los diseños de políticas culturales de cada gestión; y por último esperamos contribuir con un documento inicial que posibilite futuros análisis de un área poco investigada hasta el momento.

\section{Metodología de relevamiento}

Este rastreo abarca el Gran Buenos Aires (GBA) ${ }^{4}$. Tomamos aquellos coros que articulan la formación musical a partir de la práctica coral con la inclusión social . El rastreo identifica el escenario existente desde el 2016 al 2018. Por ende, se muestran comparativamente los coros que funcionaban en cada año, reparando en sus cambios de articulaciones, o si continuaron funcionando o no. También es importante aclarar que hay una serie de emprendimientos que presentan ambigüedades a la hora de definir si se trata de proyectos de inclusión; en muchos casos depende de la acepción que se maneje. Tomamos como elemento definitorio la auto-adscripción, es decir, si quienes desarrollan el proyecto lo consideran de inclusión social o -en caso de que no tomen ese término- lo identifican como enfocado en poblaciones en situación de vulnerabilidad. 
El rastreo de los diferentes proyectos o programas de coros infantiles y juveniles que se despliegan en esta área requirió de una estrategia metodológica que combina:

- Rastreo de medios de comunicación tales como periódicos, revistas, sitios Web de diferentes organismos de gobierno (nacional, provincial y municipal), y páginas web, blogs o redes sociales de proyectos, programas y fundaciones, entre otras.

- Entrevistas semi-estructuradas a responsables y docentes de los proyectos corales.

Desde esta estrategia metodológica vislumbramos distintas variables que utilizamos para estructurar el corte de nuestro campo. Esas variables son: nombre del coro, locación, año de creación, dependencia institucional, objetivos, población destinataria y repertorio, entre otras. En el siguiente apartado proponemos una primera aproximación al campo desde una perspectiva cuantitativa para luego profundizar y complejizar el análisis desde un abordaje más cualitativo.

\section{Aproximaciones analíticas}

Este apartado propone un abordaje de los proyectos corales donde se articulan los datos relevados que arrojan información numérica con una aproximación a esos datos desde las interpretaciones que los distintos actores hacen de los mismos. De esta manera veremos cómo se complejiza y completa una investigación en la cual se revelan tan importantes los números como sus significaciones e implicancias en el mundo social que pretenden intervenir e impactar.

\section{1- Desde una perspectiva descriptiva}

En el 2016 se realizó la primera aproximación al campo a través de un rastreo por internet, con el objetivo de obtener un mapeo del universo referido. A partir de los datos arrojados en ese primer rastreo, comenzamos un análisis descriptivo donde los datos expresados nos permitieron dar cuenta del conjunto de variables mencionadas en el apartado anterior. A continuación se muestra un cuadro comparativo que refiere solo a la cantidad de coros por año, todos dependientes de la gestión pública.

Los datos relevados desde 2016 a 2018 no demuestran un cambio significativo en cuanto a la cantidad de coros, aunque sí existe una diferencia en relación a quienes son los agentes estatales de los cuales dependen.

En el año 2016 el Programa Nacional de Orquestas y Coros Infantiles y Juveniles del Bicentenario todavía nucleaba casi con exclusividad la mayoría de las propuestas de coros infantiles y juveniles del GBA, siendo menor la cantidad de coros de dependencia únicamente municipal y muy escasos los proyectos que pertenecían al Programa Provincial Orquesta Escuela. En la Ciudad de Buenos Aires, desde el año 2013 venía funcionando 
el Programa Orquestas y Coros para la Equidad, dependiente del área socioeducativa del Ministerio de Educación de esa Ciudad. Con el cambio de gestión, a fines del 2015, esta iniciativa no sufrió cambios significativos. En esta ciudad no cambió el partido político con las elecciones, Horacio Rodríguez Larreta, hasta entonces vice jefe de gobierno, fue electo. Sin embargo, a nivel nacional y provincial como en algunos municipios el resultado de las elecciones implicó un cambio de partido político en el Gobierno.

\begin{tabular}{|c|c|c|c|}
\hline \multicolumn{1}{|c|}{ Año de relevamiento } & $\mathbf{2 0 1 6}$ & $\mathbf{2 0 1 7}$ & $\mathbf{2 0 1 8}$ \\
\hline $\begin{array}{c}\text { Nombre del Programa proyecto corales } \\
\text { Programa Nacional Coros y 0rquestas del Bicentenario }\end{array}$ & 24 & 21 & 16 \\
\hline $\begin{array}{c}\text { Programa Provincial orquesta- escuela } \\
\text { Programa de Coros y 0rquestas (2018) }\end{array}$ & 1 & 1 & 6 \\
\hline Coros de gestión Municipal' & 9 & 9 & 3 \\
\hline Programa orquestas y oros para la equidad & 3 & 3 & $\mathbf{3 5}$ \\
\hline Totales & $\mathbf{3 7}$ & $\mathbf{3 4}$ & \\
\hline
\end{tabular}

Tabla 1

No obstante, los datos recolectados en 2016 no revelaron una baja abrupta en el número de coros, según información brindada por los diferentes referentes nacionales, provinciales y municipales. El relevamiento del año 2017 muestra una pequeña variación en el número de coros que nucleaba El Programa Nacional. Los proyectos de coros dependientes de la gestión municipal y provincial se continuaron.

Es en 2018 dónde se hace significativa la redistribución de coros en cuanto a la vinculación de los órganos estatales de los cuales pasan a depender estos proyectos. Notándose modificaciones tanto en su administración como en su propuesta metodológica de enseñanza. El Programa Provincial Orquesta Escuela PPOE pasa a depender de la Dirección de Políticas Socioeducativas, y cambia su nombre a Programa Coros y Orquestas de la Provincia de Buenos Aires. De esta manera no sólo asume la creación de nuevos coros en la provincia de Buenos Aires, sino que también absorbe algunos que dependían del Programa Nacional del Bicentenario. Asimismo, el Programa Nacional de Orquestas y Coros del Bicentenario sigue desarrollándose, con nuevas directivas y nuevas formas de gestión. 
Observamos entonces, que es en el 2018 cuando el cambio de orientación de las políticas culturales públicas se empieza a plasmar en concreto tanto en la reorganización y redistribución administrativa como en la metodología de enseñanza que se comienza a implementar en los mencionados proyectos corales dentro del GBA.

Si bien entendemos que no se agota aquí el análisis, buscamos aproximarnos a la problemática que encontramos clave para visibilizar los procesos transicionales propios de los cambios en los diseños, la planificación e implementación de la política cultural ${ }^{8}$ en cada gestión pública.

\section{2- Desde una perspectiva etnográfica}

\section{a. De la centralización a la federalización de los recursos públicos}

De acuerdo a nuestro sistema federal de gobierno, tanto los gobiernos de la provincia como de los municipios, cumplen funciones relativas a la política cultural y a la administración de asuntos culturales de la comunidad, dentro de sus respectivos ámbitos territoriales o comunales. Este tipo de administración se puede instrumentar con acciones que promuevan políticas descentralizadas o, por el contrario, en ocasiones se pueden instrumentar políticas que tiendan a la centralización de políticas culturales (Harvey, 1977).

En este sentido, encontramos que varios de nuestros entrevistados, quienes ocuparon y ocupan cargos de coordinación de los Programas mencionados -en sus distintos niveles-, coinciden en afirmar que a inicios del 2016 las autoridades del Ministerio de Educación deciden "Federalizar" el Programa Nacional de Orquestas y Coros del Bicentenario.

Puntualmente a mediados de 2018, quién había ocupado el cargo de coordinador del Programa Provincial de Orquesta -Escuela al tiempo que era el referente del Programa del Bicentenario en Provincia de Buenos Aires, nos comentaba que en el 2016 el Estado Nacional decide que el Programa Nacional de Coros y Orquesta se federalice. Destacando que este hecho tenía su lado bueno y su lado malo.

Este cambio de orientación de política tanto educativa como cultural analizada desde su diseño e implementación implica una batería de acciones que puestas en marcha revelan la tensión entre el paso de una política centralizada a otra descentralizada.

Esta tensión se refleja en las propias reflexiones de uno de los docentes:

Pero en la Provincia está el hecho de que la federalización generó esto también. ¿Qué sentido tiene tener un equipo de coordinación nacional en tanto y en cuanto no vaya a haber lineamientos a nivel nacional, como lo había antes? Esto promueve una enorme desigualdad. Porque lo que sucedía desde la coordinación nacional es que se trataba de equiparar, que todos tuvieran lo que necesitaban, o sea, si los coros del sur necesitaban un salario mayor para sostener un nivel de vida que es el que tienen en el sur se gestiona desde ahí; sí 
había que meter más recursos en el NOA o en el NEA por tal o cual razón se hacía, si ...Se iba midiendo.(...) Ahora vas a tener provincias que tengan un coordinador que abogue por el cuidado del Programa y que entiendan la fundamentación que tenían, y que siga con esos lineamientos, y algunos que no, $\mathrm{y}$ algunos que sean absolutamente funcionales a los gobiernos provinciales $\mathrm{y}$ decidan frenarlos, o destruirlos, o transformarlos a gusto y piaciere." (Docente de coros del Programa de orquestas infantiles y juveniles en Ciudad de Buenos Aires, Diciembre de 2016, Buenos Aires).

A raíz de los comentarios podemos inferir como antes de la llamada "federalización" el Programa Nacional, se caracterizaba por centralizar las acciones del Programa subrayando características como la existencia de una coordinación general central (coordinador y equipo). Esta coordinación tenía la potestad para coordinar acciones, tomar decisiones, destinar fondos para la creación de nuevos proyectos, así como realizaba la licitación y distribución de instrumentos. Asimismo llevaba a cabo la convocatoria para cubrir nuevos cargos docentes como la organización de capacitaciones y jornadas a nivel Nacional. Con la política de descentralización el marco de acción del Programa, según nos informaba el actual coordinador del Programa Nacional, se redujo a la organización de capacitaciones, espaciadas entregas de instrumentos, y algunos intercambios musicales con algunas provincias.

Como se observa en el campo y dan cuenta nuestros registros, el cambio de gestión se planteó de una manera bastante abrupta;

- A nivel administrativo-nacional se activó un desplazamiento institucional que proponía "federalizar" la actividad de orquestas y coros, pero con una escasa comunicación en cuanto a la planificación y al diseño que la nueva propuesta política de gestión pretendía implementar desde el ámbito nacional. A nivel Provincial uno de nuestros interlocutores entrevistados nos explicó que cuando él ingresó las orquestas y los coros ya tenían problemas y que por lo tanto el panorama político era, según sus palabras "muy feo". Muchos de los docentes, comentaba, recién cobraron a mitad de año.

- A nivel comunicativo se demoró la transmisión de los cambios de rumbos de forma clara y precisa de las nuevas decisiones políticas respecto a la continuidad en las actividades, al giro en las modalidades de enseñanza y principalmente en lo que refiere a la estabilidad laboral de los y las educadores/as. Situación que generó incertidumbre laboral y enojo.

Frente a este proceso de cambio, que devino en una sensación de indefinición por muchos meses, no hubo una dimensión concreta de las consecuencias que las nuevas decisiones políticas tendrían en los docentes, en los niños y niñas y jóvenes participantes; como tampoco se reparó en la salud de una política cultural estatal que, pese a sus imperfecciones, tenía un gran alcance y efectos multiplicadores de oportunidades y posibilidades. 
Si bien luego la Secretaría de Políticas Socioeducativas a través del Programa Provincial de Orquesta Escuela subsanó el bache en el proceso de transición, fue un periodo angustiante para los actores involucrados.

El registro realizado justo en este periodo (2016-2018) nos permitió, dar cuenta de la emergencia de cambios a nivel de la organización de los programas existentes, lo que nos posibilitó advertir dinámicas y orientaciones de las políticas culturales en las distintas gestiones. La nueva configuración sociopolítica que empezó a delinearse a inicios de 2016 atravesó a estos proyectos impactando en su devenir y desarrollo.

Estos movimientos permiten dar cuenta, como plantea Raggio (2013), de un "estado en movimiento". Es por ello que creemos que reflexiones como la aquí expresada nos permiten construir herramientas para pensar las dinámicas internas de las continuidades-discontinuidades de los proyectos culturales estatales. Por ejemplo, hacer foco en el modelo de enseñanza que cada gestión adoptó para implementar en las propuestas de coros y orquestas constituye un elemento más que resalta la importancia del registro sistematizado. De esta forma se habilitan documentos que pueden contribuir a venideras tomas de decisiones a la hora de pensar nuevas propuestas de intervención social desde la gestión pública.

\section{b. Modelos de enseñanza en disputa}

Como consecuencia del cambio de gestión a nivel administrativo, uno de los ejes centrales que sufrió modificaciones fue el referido a los modelos de enseñanza. Particularmente el Programa Nacional de Coros y Orquestas del Bicentenario venía trabajando con una propuesta de enseñanza colectiva, la cual se vio afectada por la influencia de la dinámica que adoptó la gestión provincial. Esta se reemplazó por un sistema o modelo tendiente a un trabajo más personalizado, de "uno a uno".

Los docentes del programa de Bicentenario venían trabajando con un diseño que proponía un modelo de enseñanza musical colectiva, como consta en los documentos e informes que realizaron referentes del programa como Marcelo Zanelli. En el artículo ¿De qué hablamos cuando hablamos de inclusión? Orquestas infantiles y juveniles", Zanelli manifiesta cual es la esencia del modelo de enseñanza colectiva:

En otras palabras, sin negar la necesidad de la práctica, la técnica y el estudio en solitario, la enseñanza colectiva habilita otro tipo de intercambios y es la base de una forma de vincularse con la música y con los "otros" que potencia la cooperación y el trabajo en conjunto”. (Zanelli, 2014, p.146).

Este modelo no supone dejar de lado la calidad educativa o la idea de inclusión, como queda expresado en el mismo artículo;

Desde el inicio, el proyecto puso en el centro de la tarea una idea de inclusión educativa con calidad. Es decir, concentrando la acción de educar en aquello que se oferta más allá de la demanda. En tanto se trata de un proyecto orquestal (no hay pedagogía sin proyecto), recuperar el valor de la cooperación sobre la 
base de un intercambio en el cual los participantes obtienen beneficios de un encuentro que requiere el desarrollo de una habilidad que excede ampliamente el campo de la música, realza la importancia de los espacios en los que los intercambios no sólo tienen el potencial de mejorar los rendimientos escolares, la permanencia y terminalidad de la educación obligatoria, sino también y en una misma operación, restituyen la posibilidad de poner en práctica eso que denominamos civilidad y ejercicio de la ciudadanía. Un espacio en el que la ciudadanía se torna una práctica construida sobre la acción y el trabajo conjunto (Zanelli, 2014, p.152).

Este modelo colaborativo, en el cual el docente o instructor cumple un rol de mediador entre los contenidos que deben trabajarse en los grupos, deja de lado la lógica de lo individual, de concepciones que tienen que ver con el esfuerzo o mérito personal, como lo han expresado aquellos docentes que trabajaron con esta metodología desde los inicios del programa hasta iniciado el año 2016.

La enseñanza colectiva plantea la necesidad de mantener la atención sobre el grupo al tiempo que sobre el recorrido de cada integrante, en agrupamientos en algunos casos fluctuantes y mayormente heterogéneos. En este sentido, se trata de experiencias de convivencia "democrática" que ponen en cuestión la fragmentación social y educativa dominante. Y si bien son altamente valoradas por adultos y jóvenes, plantean desde el punto de vista de la enseñanza, la necesidad de encontrar respuesta a problemas de nivel técnico-pedagógico, desde el inicio. (Finnegan y Serulnikov, 2015, p.32)

Desde una dinámica de enseñanza-aprendizaje colectiva, las dificultades emergentes surgen y se resuelven dentro del grupo. De esta manera, se pone en valor la experiencia en favor del colectivo, esa experiencia intransferible se hace visible en el momento de la acción, en el momento de la práctica (Larrosa, 2006).

El modelo de enseñanza que propuso la nueva gestión del programa provincial ${ }^{10}$ apuntó a un aprendizaje más personalizado, ligado al modelo tradicional de enseñanza de la músi$\mathrm{ca}$, docente-alumno. Con esta metodología se propone atender las necesidades puntuales y las trayectorias diversas de cada miembro.

El ex-coordinador del Programa Provincial y del Bicentenario en provincia nos comentaba que antes había una política como de todo grupal y nada individual. Asimismo agregaba que lo grupal sirve para una actividad grupal, como podría ser el coro pero que para las orquestas la enseñanza colectiva no era suficiente dado que los instrumentos necesitan de la práctica individual.

Esto no implica que se desestime la práctica musical colectiva como forma de aprendizaje, ya que los ensayos del coro y las presentaciones grupales son una instancia de aprendizaje en sí misma, aún así dicho modelo mantiene la mirada individual, atendiendo y detectando las particularidades y diferencias en de cada miembro del coro. Una mirada personalizada se 
entiende como el acceso a una educación común para el conjunto de aquellos jóvenes, niños y niñas que acceden a la práctica musical coral, definiendo contenidos que implican cierta transversalidad y garanticen el mismo acceso al conocimiento (Diker, 2008).

Así las cosas, las intenciones corales de nuestro interlocutor recién mencionado, giraba en torno de organizar una propuesta de coro-escuela. De acuerdo a sus explicaciones la misma consistía en ir separando a los niños por niveles, de acuerdo al grado de afinación. De este modo vas incluyendo a cada niño en el nivel que corresponde. Nos explicaba que así se va armando la escuela coral, con un pre-coro, un coro y el coro juvenil.

Cabe aclarar que este es un modelo utilizado por las orquestas y coros nucleados en el Sistema de Orquestas Infantiles y Juveniles de Argentina (SOIJAR) conocido como pedagogía Orquesta Escuela (que fue impulsado en Venezuela con el Sistema Orquesta Escuela y tiene su desarrollo local desde 1998 en la Orquesta Escuela de Chascomús).

Si bien en la práctica se vislumbran las tensiones y contrariedades que implican la implementación de una enseñanza musical que requiere tanto el esfuerzo colectivo como el individual. El desafío se complejiza cuando se vincula cada método de enseñanza con el objetivo de inclusión que ambos persiguen.

Respecto a esta tensión un docente nos comentaba "Un proyecto de inclusión con coros no se si puede partir desde vengan todos y cantemos". Y continuaba “...o sea, para que funcionemos como un proyecto de inclusión, tenemos que funcionar como coro. A si no estamos incluyendo a nadie, o sea, como que es un aguantadero de pibes". (Docente del Programa de orquestas infantiles y juveniles de la Ciudad de Buenos Aires, Diciembre de 2016)

El intercambio con nuestros interlocutores nos invita a pensar en las complicaciones que lleva implementar un proyecto que prioriza lo colectivo o lo individual y que a la vez persiga objetivos de inclusión. Sobre todo cuando el concepto de inclusión no solo se presenta asociado con lo individual y lo colectivo sino que va definiendo políticas culturales, reparando poco en el carácter polisémico de término "inclusión". Es aquí donde estos conceptos aparecen en disputas, se tensionan y se significan y resinifican constantemente. La propia práctica es la que genera nuevas indagaciones y hace visible las múltiples interpretaciones del término.

Para unos incluir desde la música será a través de la calidad del proceso de la práctica colectiva. Para otros, en cambio será a través de una educación de calidad desde un trabajo que prioriza la enseñanza uno a uno. En algunos casos estos términos aparecerán juntos, en otros divorciados, en otras como dos caras de la misma moneda.

Desde el punto de vista de la mayoría de nuestros entrevistados, estos cambios en la metodología de enseñanza fueron habilitando tensiones, contradicción, acepciones y rechazos hacia el interior tanto del grupo docente como hacia y entre el equipo de coordinación. Lo que se vio traducido en peleas, denuncias, renuncias y lucha por la continuidad de estos proyectos. Debido a esta abrupta implementación de la nueva metodología, sumado a las modificaciones administrativas, en un primer momento se percibió como una discontinuidad del proyecto cristalizando un descenso en la matrícula de los coros, que con el correr del tiempo, pudo estabilizarse y que según los y las docentes entrevistados/as, tuvo nuevos ingresos a partir del 2017 aproximadamente. 
En lo que respecta entonces a este cambio en la orientación de política socioeducativa, podemos observar que los cambios fueron concretados alrededor de inicios del año 2017, y al decir de nuestros entrevistados, han sido implementados a medias, ya que si bien había una intención de modificar la metodología de la enseñanza musical, no hubo suficientes recursos económicos para llevar a cabo la designación de una mayor cantidad de docentes por coro u orquesta, con lo cual terminaron conviviendo ambos modelos de enseñanza, de acuerdo a la disposición de recursos humanos con los que contara cada agrupación. Es así que si nos proponemos analizar la transición de las gestiones en clave de diseño, infiriendo que; o hubo una ausencia del mismo o que este se planteo de forma desordenada, sin tener en cuenta factores que impactarían directamente en la población destinataria. Dejando a su suerte la complejidad que conlleva en una política pública la elaboración consciente de un plan de gestión, orientado tanto a impactar en el plano educativo como cultural de una determinada comunidad o sociedad.

Por consiguiente, quedó en manos de los y las docentes poder realizar la transición con las contradicciones disputadas de un modelo a otro, para garantizar esa educación inclusiva y de calidad que ambas gestiones han postulado como principios fundamentales en sus objetivos.

\section{El diseño de la política cultural en la gestión pública}

Cabe aclarar, que cuando hablamos de diseño lo primero que se nos viene a la mente es su relación con lo concreto, las imágenes, las estructuras, las formas que tienen diferentes objetos, entre otras.

En dos ocasiones tuvimos la oportunidad de presentar avances de esta investigación, sobre el "diseño" de las políticas culturales en el ámbito de la gestión pública. En ambas instancias fuimos interpeladas con la misma pregunta: ¿Que tienen que ver las políticas culturales con el diseño?

Avenbrug y Materrese (2019), proponen pensar la relación entre política y cultura en clave de diseño. Como sugieren las autoras, el cruce conceptual entre política y cultura motiva la reflexión sobre las disputas de sentido que se encuentran imbricadas en los distintos tipos de diseño reconociendo que ambos campos-el de diseño y el cultural- se atraviesan mutuamente no solo en el plano conceptual sino también en el plano de las prácticas.

De este modo, cuando abordamos el análisis de las distintas maneras de gestionar una misma propuesta de política cultural en el ámbito de la gestión pública es necesario identificar los lineamientos de las gestiones anteriores así como de la vigente. Esto permitirá trazar los lazos conceptuales, operativos y prácticos de manera transversal. Analizar sus conceptualizaciones, interpretaciones así como las modalidades prácticas del hacer y del pensar los objetivos que se continúan, se modifican o se eliminan. Estos elementos se constituyen en procedimientos claves para realizar una transición de una gestión a otra. El cambio de diseño se verá reflejado en la estructura administrativa y en los usos diferenciales de los conceptos utilizados. 
En este sentido, una de las primeras discusiones conceptuales que identificamos en nuestra investigación giró en torno a la dimensión referente a las competencias de la administración pública. Problemática, que como se planteó anteriormente, la ubicamos dentro de las discusiones en torno a la dicotomía entre descentralización y centralización de la cultura. El caso de las concepciones y prácticas respecto a los modelos de enseñanza y su relación con la inclusión, son ejemplo de ellos.

Finalmente, vemos cómo esas distintas maneras de ver, de concebir y de poner en práctica dejan ver entre líneas las múltiples, distintas, opuestas y hasta contradictorias maneras de pensar y hacer acciones que parecieran iguales.

Es necesario destacar que en el caso de las políticas culturales desarrolladas por el Estado las orientaciones simbólicas, las disponibilidades materiales así como lo que se recupera como cuestión socialmente problematizada se encuentra fuertemente matizada por el partido de gobierno que esté administrando el Estado (Bayardo,2000,García Canclini, 1987). Es decir, detrás de cada decisión del Estado hay personas que toman decisiones respondiendo a una ideología partidaria que, en la mayoría de los casos, se corresponde al partido del Gobierno en función. Consideramos que este detalle no es un dato menor a tener en cuenta a la hora de analizar políticas culturales que implementa la gestión pública en función.

De lo de recién analizado se desprende como se hace necesario realizar diseños de transición, ya que de esta manera se evitaría en gran medida la incertidumbre inquietante de continuidad o interrupción que estos cambios sin planificación generan.

\section{Reflexiones finales}

En el presente trabajo nos propusimos presentar un panorama aproximado existente de coros infantiles y juveniles que se desarrollan en el Gran Buenos Aires a partir del relevamiento realizado en 2016-2018. La cantidad y heterogeneidad de coros -aún en el marco de ciertos denominadores comunes- posibilita organizar el escenario existente, si bien sabemos que siempre algunos pueden quedar afuera.

No obstante, consideramos que esta es la base para futuras indagaciones que implican tanto la ampliación, profundización y actualización del rastreo, como también abordajes etnográficos mediante nuevas entrevistas en profundidad acompañadas por observación con participación en distintos proyectos de coros.

Asimismo, a partir de lo observado podemos afirmar que esta diversidad expuesta de proyectos, actores y articulaciones posibilita tanto revalorizar diferentes expresiones musicales como dar cuenta el entramado complejo de poner en práctica políticas culturales en el marco de la gestión pública.

Pudimos registrar los cambios a nivel de la organización administrativa de los programas existentes, lo que nos permitió advertir, a través de distintas perspectivas, las dinámicas y reorientaciones en las modalidades de gestión. Esto nos permitió hacer foco en los métodos de enseñanza, en los conceptos utilizados, así como en los procesos de transición implicados entre el paso de una gestión a otra en el ámbito estatal. 
Desde la aproximación descriptiva pudimos obtener datos cuantificados que combinados con el trabajo etnográfico cualitativo nos permitieron abordar la investigación de manera integral al tiempo que visibilizar la importancia del registro sistematizado de esos procesos. Finalmente, la nueva configuración sociopolítica que se empieza a delinear a inicios de 2016, en el ámbito del GBA, atravesó a estos proyectos impactando en su devenir y desarrollo. Muchos de las propuestas sobre los cuales realizamos el relevamiento se encontraban insertas en un proceso de modificación interna, así como en conflictos gremiales que impactaban directamente en el desarrollo de las actividades musicales.

\section{Notas:}

1.Versiones preliminares de este trabajo fueron presentados en el $3^{\circ}$ Congreso Coral Argentino y $1^{\circ}$ Latinoamericano de OFADAC (2018) y en los Coloquios de Investigación y Diseño de la Universidad de Palermo (2018/2019). Agradecemos los valiosos y nutridos comentarios recibidos. Los mismos han enriquecido las discusiones abordadas en el presente artículo.

2. El 10 de Diciembre de 2015 y como resultado de las elecciones, el Gobierno Nacional es asumido por el nuevo presidente Mauricio Macri. El Gobierno Provincial por Marìa Eugenia Vidal y la Ciudad de Buenos Aires por Horacio Rodríguez Larreta, quién renovó su mandato. En esta oportunidad se dio que los niveles de administración recièn mencionados se encontraban alineados partidariamente. Es decir, los tres candidatos respondian al Frente Cambiemos, de perfil neoliberal. El Gobierno que dejaba su mandato tanto a nivel Nacional como Provincial pertenecían al Frente para la Victoria, partido alineado a la ideología peronista.. Lo cual el cambio de Gobierno significó un cambio de rumbo significativo a nivel tanto ideológico, traducido en las orientaciones de sus políticas publicas. Ejemplo de ello son los proyectos de coros infantiles y juveniles que estudiamos en este trabajo.

3. Grupo de Investigación sobre Música e Inclusión, radicado en la Universidad de Avellaneda. Este grupo está integrado por investigadoras formadas y en formación provenientes de distintas disciplinas como antropología, ciencias políticas, periodismo, dirección coral, sociología y gestión cultural

4. Gran Buenos Aires incluye Ciudad Autónoma de Buenos Aires y los 24 partidos que integran el primer cordón de la Provincia de Buenos Aires.

5. Es necesario aclarar que el concepto "inclusión social” más allá de tener múltiples acepciones refiere tanto a la dimensión material como a la simbólica. Varios autores han analizado el concepto en relación a su contraparte exclusión (Castel,1997; Perez Rubio, 2006; Belfiore, 2002; etc.) y han problematizado y complejizado el contexto de sus diferentes usos (Avenburg, Cibea, Talellis, 2019) 
6. El Programa Provincial cambió su denominación en 2018, esta denominación está estrechamente ligada a la inclusión de formaciones corales en el programa que hasta el momento sólo nucleaba orquestas.

7. Estos coros no no pertenecen a programas específicos, aunque en ocasiones puedan articular con ellos. La característica principal es que son financiados por los municipios y que eran coros que existían antes de la formación de los programas socioeducativos, aunque como ya mencionamos, algunos posteriormente generaron articulaciones con los programas existentes. Se desarrollan en los siguientes municipios: Berazategui, Esteban Echeverría, Hurlingam, Lomas de Zamora, Morón, San Isidro.

8. Cabe aclarar que por política cultural entendemos de acuerdo a Garcia Canclini (1987 p, 26) "el conjunto de intervenciones realizadas por el Estado, las instituciones civiles y los grupos comunitarios organizados a fin de orientar el desarrollo simbólico, satisfacer las necesidades culturales de la población y obtener consenso para un tipo de orden o de transformación social"

9. Cabe aclarar que esta gestión absorbió gran número de coros que pertenecían al Programa Nacional de Bicentenario, y que por tal motivo se vieron en la necesidad de adecuar sus modelos de enseñanza musical, y adoptar las diseñadas por el Programa Provincial de Orquesta Escuela.

10. El programa PPOE ya venía desarrollando este modelo de enseñanza en las orquestas que nucleaba.

\section{Bibliografía}

Antelo, E., Zanelli, M. (2004). Informe Final Orquestas infanto/juvenil (Lugano) Dirección de Investigación Secretaría de Educación. Ciudad Autónoma de Buenos Aires. Disponible en https://www.google.com/url?sa=t\&rct=j\&q=\&esrc=s\&source=web\&cd=1\&ved=2ahU KEwizsY-mm6vkAhVELLkGHc6HAlUQFjAAegQIABAC\&url=http\%3A\%2F\%2Fwww. buenosaires.gob.ar\%2Fsites\%2Fgcaba\%2Ffiles\%2Flibro_educacion_en_caba_orquestas. pdf\&usg=AOvVaw3U5uKR3My86I3pA_PjK02H

Avenburg, K., Cibea,A. y Talellis, V. (2017). Las orquestas infantiles y juveniles del Gran Buenos Aires. Estudio descriptivo del panorama de proyectos y programas vigentes entre 2014 y 2015. Foro de educación musical, arte y pedagogía 2(2), 13-57.

Avenburg, K, Cibea, A, Talellis, V. (2019). Las Artes frente a la exclusión. Manifestaciones artísticas como prácticas de inclusión, integración y /o transformación social - 1a ed. Avellaneda : Undav Ediciones.

Bayardo, R. (2000). Antropología, Identidad y Políticas Culturales. En NAYA (Noticias de Antropología y Arqueología). Disponible en. http: //www.naya.org.ar/articulos/identi01.him/

Belfiore, E. (2002). "Art as a means of alleviating social exclusion: Does it really work? A critique of instrumental cultural policies and social impact studies in the UK". En International Journal of Cultural Policy 8 (1): 91-106.

Castel, R. (1997). La metamorfosis de la cuestión social. Paidós: Buenos Aires 
Diker, G. (2008). ¿Cómo se establece que es lo común?. En Frigerio G. y Diker G. (edit.) Educar: posiciones acerca de lo común (pp..147-157) Buenos Aires, Del Estante.

Finnegan, F. Serulnikov, A. (2015) La enseñanza y el aprendizaje como ejes de la propuesta socioeducativa. El Programa Nacional de Orquestas y Coros Infantiles y Juveniles para el Bicentenario. Ministerio de Educación de la Nación. Dirección Nacional de Información y Evaluación de la Calidad Educativa (DiNIECE)

Mercado, C. (2019). En reversa la mirada y en futuro el corazón: teatro comunitario y disputas en torno al arte para la transformación social. En J. Infantino (edit.), Disputar la cultura. Arte y transformación social (pp..93-132) Caseros, RGC Libros.

Moyano, M (2019). Creación, gestión e institucionalización de una organización socioartística: El caso de ph 15. En J. Infantino (edit.), Disputar la cultura. Arte y transformación social (pp..93-132) Caseros, RGC Libros.

Muiños de Britos, S.M. (2010)b. La práctica musical colectiva. Aprendizaje artístico y social. Revista Iberoamericana de Educación $N^{\circ} 52$ (2). Disponible en: http://www. rieoei.org/ deloslectores/3589Muinos.pdf/

García Canclini, N. (1987). Políticas culturales y crisis de desarrollo: un balance latinoamericano. En García Canclini (ed.), Política culturales en América Latina (pp. 13-61). México, Grijalbo.

Frith, S. (2001). "Hacia una estética de la música popular”. En Cruces, Francisco y otros (eds.), Las culturas musicales. Lecturas de etnomusicología. Pp. 413-435. Madrid: Trotta.

Harvey, E. (1977). "La política cultural en Argentina". En Políticas culturales: estudios y documentos. Unesco. Madrid

Infantino, Julieta. (2008). "El arte como herramienta de intervención social entre jóvenes en la ciudad de Buenos Aires. La experiencia de 'Circo Social del Sur". En Andrea Tammarazio (Editora responsable) Medio Ambiente y Urbanización $N^{\circ}$ 69. Niños, niñas y jóvenes como agentes de cambio. Buenos Aires: Instituto Internacional de Medio Ambiente y Desarrollo, IIED- América Latina. Pp. 35-54.

Larrosa, J. (2006) La experiencia y sus lenguajes.Conferencia. En la Formación Docente entre el siglo XIX y el siglo XX. Serie Encuentros y seminarios. Pp 1-11. Disponible en https://www.google.com/url? sa=t\&rct=j\&q=\&esrc=s\&source=web\&cd=1\&ved=2ahU KEwj35PX-lavkAhV6H7kGHa7ABL0QFjAAegQICBAC\&url=http\%3A\%2F\%2Fwww. bnm.me.gov.ar\%2Fgiga1\%2Fdocumentos\%2FEL001417.pdf\&usg=AOvVaw1lsh132K GMG6vSHyrTFWpj

Lenger, D. (2013). "El coro como herramienta de transformación social". Disertación en el $1^{\circ}$ Congreso Coral Argentino. Mar del Plata, 20 a 23 de Junio.

Oszlak, O. (2009). "Implementación participativa de políticas públicas: aportes a la construcción de un marco análitico". En A. Belmonte (et.al.). Construyendo confianza. Hacia un nuevo vínculo entre el Estado y Sociedad Civil, Volumen II 8pp. 9-48). Buenos AIres, Centro de implementación de Políticas Públicas para la Equidad y el Crecimiento (CIPPEC) y Subsecretaría para la Reforma Institucional y Fortalecimiento de la Democracia, jefatura de Gabinete de Ministros, Presidencia de la Nación.

Pérez Rubio, A.M. (2006). "Acerca de la exclusión y otras cuestiones próximas". Revista de Estudios Regionales, y Mercado de Trabajo 2. Disponible en: http://www.simel.edu.ar/ archivos/documentos/RS2\%20Rubio.pdf 
Raggio, L. (2013). Los derechos culturales en la Ciudad Autónoma de Buenos Aires: una contienda simbólica en plena desarrollo. En Cuadernos de Antropología 10, 277-297.

Zanelli, M. (2014). Orquestas Infantiles y Juveniles ¿De qué hablamos cuando hablamos de inclusión? En Lépore, Silvia [et.al.]. - 1a ed. La Educación en la Ciudad de Buenos Aires: Aportes desde la investigación. Pp 143-154.Ministerio de Educación del Gobierno de la Ciudad Autónoma de Buenos Aires

\section{Otras fuentes}

Dirección Provincial de Políticas Socio Educativas. Coros y Orquestas. http://abc.gov.ar/ socioeducativa/programas/coros-y-orquestas

Fundación Sistema de Orquestas Infantiles y Juveniles de Argentina. https://www.sistemadeorquestas.org.ar/

Programa Nacional de Orquestas y Coros Infantiles y Juveniles. https://www.argentina.gob. ar/educacion/gestioneducativa/orquestas-coros

Programa Nacional de Orquestas y Coros Infantiles y Juveniles para el Bicentenario. https:// www.educ.ar/noticias/127160/la-fiesta-de-la-musica-orquestas-y-coros-infantiles-yjuveniles-para-el-bicentenario

Abstract: From the end of the 20th century, in Argentina, different projects which proposes the arts as a tool for social inclusion started to blossom. In consequence, many authors have focused their researches about the "arts" and the social dimension of it in a group or community. Following this line of thoughts, our goals are focused on children and youth choirs, located in Gran Buenos Aires (the suburban area) and managed by the State. From the survey developed during 2016-2018, we present a depiction, from a descriptive-comparative perspective of the obtained data, about some aspects that are highlighted topics in the dispute for the meanings of doing, involved in the designs of cultural politics of every administration.

Keywords: children and youth choirs - ways to teach - social inclusion - public administration - cultural design

Resumo: A partir do final do século XX, na Argentina, propostas que instalam a arte como ferramenta de inclusão social começam a se multiplicar. Consequentemente, vários autores orientaram suas pesquisas refletindo sobre o lugar da "arte" e sua dimensão social em um grupo ou comunidade. Seguindo essa linha, nossas pesquisas se concentram nos coros de crianças e jovens, da Grande Buenos Aires, administrados pelo Estado. Com base na pesquisa realizada no período 2016-2018, propomos refletir, de uma perspectiva des- 
critivo-comparativa dos dados coletados, sobre alguns aspectos vislumbrados na disputa sobre os sentidos do fazer, envolvidos na elaboração de políticas. cultural de cada gestão.

Palavras chave: coral infantil e juvenil - métodos de ensino - inclusão social - gestão pública - desenho cultural

[Las traducciones de los abstracts fueron supervisadas por el autor de cada artículo] 